\title{
Intercellular contacts between chick stereocilia after acoustic overstimulation $^{1}$
}

\author{
Yehoash Raphael *, Yu Wang, Michael K. Lee \\ Kresge Hearing Research Institute, The University of Michigan, 1301 East Ann Street, Ann Arbor, Michigan 48109-0506, USA
}

(Received 15 April 1993; Revision received 9 September 1993; Accepted 2 October 1993)

\begin{abstract}
The goal of this study was to analyze the distribution of actin and the shape of stereocilia of chick hair cells that survive acoustic trauma. Chicks were exposed to intense octave band noise for $4 \mathrm{~h}$. They were killed either immediately after the exposure, after 6 or after $72 \mathrm{~h}$. The basilar papillae were examined using scanning electron microscopy and fluorescence microscopy, with phalloidin as an actin-specific probe. Injured hair cells which survived the trauma displayed disorganized stereocilia bundles, elongated stereocilia, and supernumerary stereocilia bundles. Tips of stereocilia in the damaged region of the basilar papilla appeared to be in contact with tips of stereocilia of neighboring hair cells. These contacts may represent 'stress links' which appear in traumatized hair cells. These results show that substantial changes in stereocilia occur within hours of exposure to intense noise. We speculate that surviving hair cells may play a role in the process of repair of the basilar papilla after noise trauma and that the changes in stereocilia structure described here are related to this role.
\end{abstract}

Key words: Chicks; Noise; Stereocilia; Regeneration; Actin

\section{Introduction}

Exposing the inner ear to intense acoustic stimuli typically results in functional and structural hearing trauma. The structural manifestation of acoustic trauma in the sensory epithelium usually involves injury or loss of hair cells (for reviews, see Saunders et al., 1991; Slepecky, 1986). Degenerating auditory hair cells are replaced by irreversible scars in mammals or new hair cells in non-mammalian species. The cellular mechanism which underlies hair cell death due to acoustic overstimulation is not known. Moreover, it is not clear why some hair cells in a damaged region degenerate while others survive. To better understand these issues, it is necessary to distinguish between injury that leads to cell death and injury that is compatible with survival and, possibly, recovery. It is also important to determine whether hair cells that survive acoustic trauma

\footnotetext{
* Corresponding author. Fax: (313) 764-0014.

1 Presented, in part, at the First Meeting on the Molecular Biology of Hearing and Deafness. La Jolla, CA, May 1992.
}

play specific roles in addition to their regular function as mechano-electrical transducers.

Actin is a conspicuous molecule at the apical pole of hair cells, present at high concentration in three different domains: stereocilia, cuticular plate and adherens junctions (Drenckhahn et al., 1985; Flock and Cheung, 1977; Raphael and Altschuler, 1992; Slepecky and Chamberlain, 1982; Tilney et al., 1983). Because of its marked presence in these structures, actin is a very useful probe for the structural analysis of healthy and traumatized hair cells (Raphael, 1991; 1993). The actin cytoskeleton of hair cells has been shown to be altered due to acoustic trauma in the lizard, chick and mammalian ear (Cotanche et al., 1987; Raphael and Altschuler, 1991; Tilney et al., 1982) and to undergo reorganization associated with hair cell degeneration and scar formation (Raphael and Altschuler, 1991; 1992).

The goal of this work was to analyze the structure of chick hair cells that survive acoustic trauma, with emphasis on the shape and organization of stereocilia. The shape and size of stereocilia are of interest because they have been shown to be altered after noise 
exposure using scanning electron microscopy (SEM) and light microscopy (LM) (Cotanche et al., 1987; Liberman and Beil, 1979; Raphael, 1991; Robertson and Johnstone, 1980). Moreover, Liberman and Beil
(1979) have demonstrated that the orderliness of stereocilia closely correlates to the threshold of neura] responses in noise-damaged cat inner ears. Finally, stereocilia are clearly observable using molecular label-

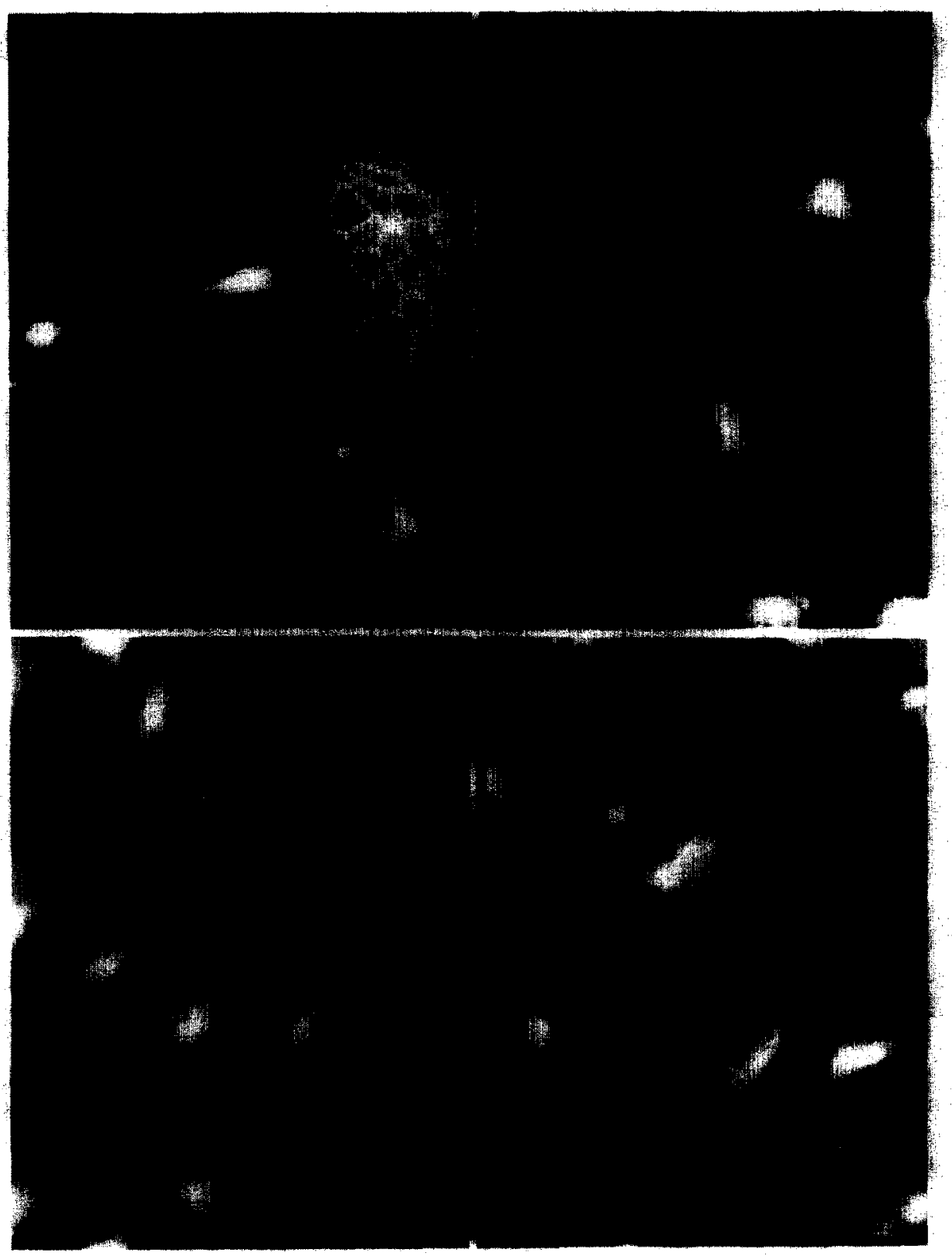

Fig. 1. Surface view fluorescence micrographs of whole mount preparations of the chick basilar papilla showing the distribution of actin filaments after a 4-h noise exposure. (A): Immediately after the exposure, an area with severe hair cell loss is observed in the short hair cell region. Expanded surfaces of supporting cells (s) fill spaces previously occupied by hair cells. Few hair cells are present in the tissue and display elongated stereocilia (curved arrows). Elongated stereocilia are in contact with stereocilia of neighboring hair cells at the tips of their hundles (arrows). (B): Cells with elongated stereocilia are also observed $6 \mathrm{~h}$ after the noise exposure (results not shown) close to the center of the lesion. Many hair cells remain in this area, but their stereocilia are disorganized. Linked bundles from adjacent cells are clearly observed (arrows). Some surviving hair cells have ectopic bundles of stereocilia. (C): An area with no apparent hair cell loss, at the periphery of the lesion, where elongated stereocilia (arrow) are present on most hair cells $6 \mathrm{~h}$ after the noise exposure. Note that supporting cells in this area do not have expanded surfaces (open arrow). (D): A region in the periphery of the lesion consisting of surviving short and tall hair cells, 3 days after the noise exposure. Disorganized bundles of stereocilia, multiple ectopic bundles and tip-tip contacts (arrows) between elongated stereocilia are observed in most cells. Bars, $5 \mu \mathrm{m}$ in $\mathrm{A}$ and in D (for B-D). 
ing techniques in chicks (Raphael, 1991; 1992) and mammals (Raphael and Altschuler, 1992; Avinash et al., 1993).

Noise-exposed basilar papillae were analyzed with SEM and fluorescence microscopy, using phalloidin as an actin-specific probe. Hair cells that were injured by noise exposure (but that did not degenerate) displayed structural changes including curved stereocilia, disorganized stereociliary bundles, elongated stereocilia and supernumerary stereociliary bundles. Contacts were often found between the tips of stereocilia of different hair cells.

\section{Materials and methods}

Ten-day old white Leghorn chick hatchlings were exposed to an octave band noise with a center frequency of $1.5 \mathrm{kHz}$ and intensity of $118 \mathrm{~dB}$ SPL for $4 \mathrm{~h}$. The exposure setup was identical to that previously described (Raphael, 1992). The data presented here are from chicks killed immediately after the noise exposure $(N=5), 6 \mathrm{~h}$ after termination of the noise exposure $(N=6)$ or 3 days after the exposure $(N=4)$. Four additional animals were used as controls.

Chicks were anesthetized with chloral hydrate and systemically perfused (intracardiac) with either $3 \%$ paraformaldehyde in $0.15 \mathrm{M}$ phosphate buffer at $\mathrm{pH}=$ 7.35 (for phalloidin cytochemistry) or $2 \%$ glutaraldehyde in $0.15 \mathrm{M}$ phosphate buffer at $\mathrm{pH}=7.35$ (for SEM). Temporal bones were rapidly removed for additional local fixation.

The auditory part of the inner ear was dissected free of bone and the tegmentum vasculosum was removed. The tectorial membrane was severely damaged, as previously describcd (Cotanche, 1987a; Marsh et al., 1990; Raphael, 1991) and therefore did not have to be removed.

For actin-specific labeling, tissues were permeabilized with $0.1 \%$ Triton X-100 in PBS for $5 \mathrm{~min}$ and incubated with rhodamine phalloidin (1:100, Molecular Probes, OR) for 23 seconds in a microwave oven (General Electric Spacemaker 2) (Smith, 1991). Extensive control tissues, from normal as well as noise-exposed animals, were processed in routine incubation with phalloidin, as previously described (Raphael, 1993). These controls helped determine that results obtained with microwave incubations did not differ from routine room-temperature incubations.

After rinsing, whole mount preparations were mounted in $60 \%$ glycerol in sodium carbonate buffer (pH 8.5) with $p$-phenylenediamine. Preparations were analyzed and photographed (Kodak T-max 400 film) with a Leitz Orthoplan microscope equipped for epifluorescence using a X100 oil objective.

For SEM, the basilar papillae were post-fixed in $1 \%$ aqueous osmium tetroxide for $60 \mathrm{~min}$, dehydrated in alcohol, critical point dried and sputter coated with gold. The samples were then analyzed and photographed with a AMRAY 1000B scanning electron microscope operated at 10 or $15 \mathrm{kV}$.

\section{Results}

We have previously published a detailed description of actin distribution in the normal avian basilar papilla (Raphael, 1992; 1993). Briefly, control (unexposed) basilar papilla cells contain intense actin labeling in the stereocilia and cuticular plate (terminal web) of hair cells and in adherens junctional complexes that connect all cells at the apical (luminal) surface of this epithelium. The apical surfaces of supporting cells appear as narrow bars that surround hair cells.

Immediately after a 4-h noise exposure, an area with severe hair cell loss was observed approximately $1 \mathrm{~mm}$ from the proximal (high-frequency) end of the papilla. Expanded surfaces of supporting cells replaced the missing hair cells (Fig. 1A). Several hair cells that survived the trauma and remained in the area exhibited elongated stereocilia, extending up to three times their normal length (Fig. 1A). With few exceptions. elongated stereocilia appeared to contact stereocilia of a neighboring hair cell. The contact between two stereocilia bundles occurred at the tips of the bundles. Extended stereocilia and intercellular contacts at the tips of stereocilia were found in all basilar papillae investigated, with the exception of control (unexposed) tissue.

Cells with elongated stereocilia were also observed 6 $h$ after the noise exposure (results not shown). Hair cell loss was less severe in more distal regions of the basilar papilla, at the margin of the lesion. Stereocilia on remaining hair cells exhibited disorganized bundles and many elongated stereocilia appeared linked to tips of stereocilia on neighboring cells (Fig. 1B). The stereociliary bundles of many surviving hair cells were ectopic; that is, they were located in one of the corners of the apical surface, in contrast to normal cells, where stereocilia extend from an area close to the center of the cell. Several cells had more than one bundle, with the ectopic bundles located near the major bundle or in a remote part of the apical surface (Fig. 1B).

In an area further distal to the center of the lesion, hair cell loss was not observed $6 \mathrm{~h}$ after the noise exposure (Fig. 1C). However, stereocilia on many hair cells in this area were elongated. Supporting cells in this area appeared normal and did not have expanded surfaces.

Three days after the noise exposure, many surviving cells in the periphery of the lesion exhibited disorganized bundles of stereocilia, multiple ectopic bundles and tip-tip contacts between elongated stereocilia (Fig. 
1D). The inter-stereocilia links were established between main bundles or between ectopic bundles at different locations on the cell surface. These changes in bundle morphology were found in short and tall hair cell regions. Based on the location along the neuralto-abneural axis of the basilar papilla, the cells shown in Fig. 1D include short and tall hair cells. Thus, elongated stereocilia and intercellular tip connections were found in both (short and tall) types of hair cells.

SEM analysis of the basilar papilla surface $6 \mathrm{~h}$ after the noise exposure revealed contacts between hair cells via extensions from the tips of stereocilia bundles (Figs. $2 \mathrm{~A}$ and $2 \mathrm{~B}$ ). The links usually connect two hair cells, but in some cases, three interconnected cells are also

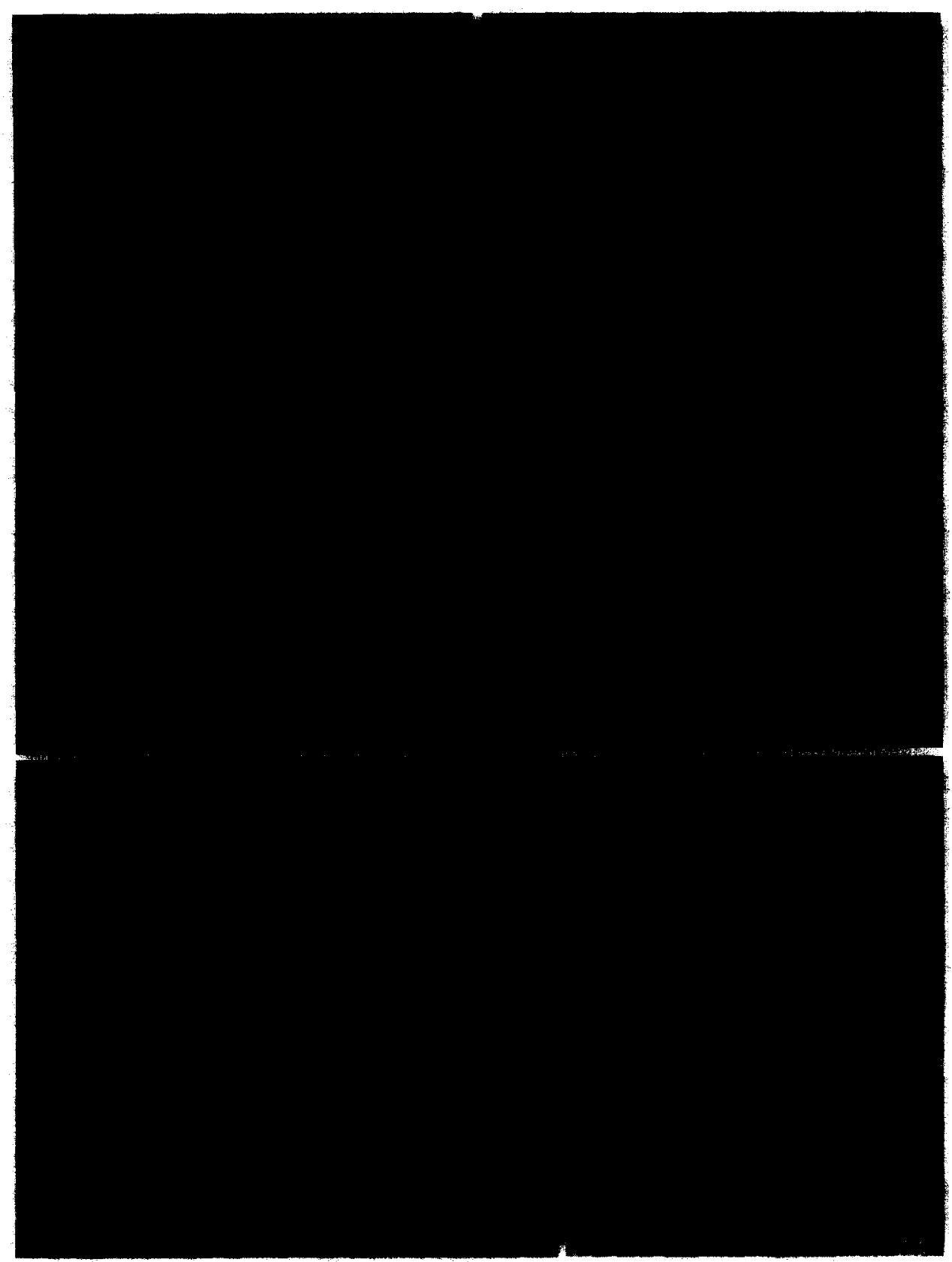

Fig. 2. SEM micrographs showing the surface of the basilar papilla $6 \mathrm{~h}$ ( $\mathrm{A}$ and $\mathrm{B}$ ) or 3 days (C and D) after the noise exposure. (A): Surviving hair cells (h) are surrounded by slightly expanded surfaces of supporting cells (s). Extensions from the tips of stereocilia bundles make a contact between two hair cells (arrow). Note that linked bundles appear relatively normal. (B): One hair cell is linked to two adjacent hair cells, one on every side (arrows). Supporting cells between linked cells may be expanded (s) but often appear normal (curved arrow). Some hair cells have elongated stereocilia with no contacts (open arrows). (C): A giant hair cell survived the trauma in the center of the lesion. The giant cell has several ectopic bundles of small stereocilia (arrows) at different locations on its apical surface. No other hair cells are observed in close proximity to the giant cell. (D): In the periphery of the lesion, a cell (center) with a reorganized bundle has neither elongated stereocilia nor tip interconnections. The cell borders normal appearing hair cells (curved arrow). Bars, $2 \mu \mathrm{m}$. 
observed (Fig. 2B). Supporting cells between interlinked hair cells are often expanded but, at other times, may appear normal (Fig. 2B). In several cases, bundles with a relatively normal appearance and location were linked to each other (Fig. 2A and B).

In the center of the lesion, some surviving hair cells had a much larger apical surface than normal cells. These 'giant' cells usually had several ectopic bundles of small stereocilia at ditferent locations on their large apical surface (Fig. 2C). Typically, a giant hair cell was surrounded by several supporting cells, with no neighboring hair cells in close proximity.

Cells with reorganized or extranumerary stereocilia also appeared in the periphery of the lesion 3 days after the trauma (Fig. 2D). These cells often neighbored normal appearing cells. Cells with reorganized bundles often, but not always, had elongated stereocilia and tip inter-connections.

\section{Discussion}

The results of this study provide evidence that hair cells that survive acoustic trauma and remain in the lesion or near the lesion reorganize the morphology of their luminal projections. This reorganization occurs within hours of acoustic overstimulation and includes relocating stereocilia, elongating stereocilia and forming hair cell-hair cell contacts at the tips of stereocilia.

Changes in the structure of stereocilia are among the earliest detectable pathologies associated with acoustic trauma in mammals (see reviews by Saunders et al., 1991; Slepecky, 1986). In chicks and mammals, some hair cells degenerate immediately after trauma, while other cells with stereociliary abnormalities can be found days or even weeks after the trauma, suggesting that these changes are compatible with cell survival (Adler et al., 1992; Liberman and Beil, 1979; Thorne et al., 1984). Abnormal stereociliary structure can be manifested as elongated stereocilia, disorganized bundles, fused stereocilia, fractured stereocilia or irregularly numbered stereocilia in mammals (Engström et al., 1983; Robertson, 1982) and chicks (Cotanche, 1987b).

Our data support the idea that stress-induced changes in stereocilia do not necessarily lead to cell death. Marsh et al. (1990) have suggested that the apical surfaces of pathologically shrunk hair cells can expand and return to normal dimension several days after acoustic trauma. Cotanche (1987b) observed surviving hair cells with damaged stereocilia 10 days after severe overstimulation with pure tone. Severely damaged stereocilia have also been found on hair cells that survive many days after acoustic trauma in mammals (Engström et al., 1983; Robertson, 1982), supporting the notion that altered stereociliary structure is com- patible with survival, and possibly repair, in mammalian hair cells.

It is not possible to determine unequivocally that the links observed with SEM contain actin. For example, these links may represent remnants of the tectorial membrane. Nevertheless, histochemical analysis enables us to identify actin in the elongated extensions that established the intercellular links. Thus, data obtained with fluorescence microscopy provide direct evidence that the elongated intercellular links contain actin and are not remnants of the degenerated tectorial membrane. Based on the appearance of these links between stereocilia of neighboring cells following acoustic trauma, we tentatively propose to name them 'stress links'.

Intercellular stress links between stereocilia have not been reported to appear in previous studies on the effects of noise on the avian basilar papilla. It is possible that SEM preparation techniques disrupt most of these fragile contacts. Perhaps our method for histochemically processing the tissue allows preservation of the links because, tissue preparation for phalloidin labeling, involves no osmication, dehydration or critical point drying. Therefore, shrinking of the biological material is minimized and other preparation artifacts are reduced. This may explain why stress links were observed in this study in all traumatized basilar papillae that were analyzed using histochemistry on whole mounts and only in some of the SEM samples.

Two arguments lead us to believe that stress links are not accidental contacts between extended stereocilia. First, like the rest of the hair cell surface, the surface of stereocilia is covered with a negatively charged glycocalyx (Santi and Anderson, 1987). As a result of this negative charge, cells or cellular extensions, such as stereocilia, are prevented from adhering to each other, unless a specialized adhesive mechanism is employed. Flock et al. (1977) have presented evidence for a negative charge on stereocilia. With a coat of negatively charged glycocalyx, it is unlikely that links between stereocilia of adjacent cells are a random accidental occurrence. Second, if the contacts between elongated stereocilia were accidental, only a small portion of extended stereocilia would be expected to participate in forming such contacts. The findings that most extended stereocilia are involved in intercellular contacts suggest that these contacts are actively established between neighboring cells.

Contacts between stereocilia of adjacent hair cells were observed and reported previously in normal guinea pigs (Hackney et al., 1988) and in noise-exposed rabbits (Borg and Engström, 1989). A variety of structural changes have been found after relatively short noise exposures in different mammals (Avinash et al., 1993; Engström et al., 1983; Thorn et al., 1986). It therefore appears that hair cells in mammals and avians 
have certain similar characteristics in their response to loud sounds, as previously reported (Raphael and Altschuler, 1992). In chicks, altered bundle morphology is found in the periphery of the lesion where only few, if any, new hair cells are added. Nevertheless, structural recovery does occur in this area (Cotanche, 1987b; Marsh et al., 1990). It therefore seems likely that elongated stereocilia and stress links are reversible.

Elongated stereocilia were present as early as $4 \mathrm{~h}$ after the onset of noise cxposure. This rapid growth suggests that elongation is an urgent stress response. The presence of biological mechanisms that allow for assembly of actin-based structures within seconds has been demonstrated in leading edge extension in dictyostelium (Hall et al., 1988) and in the response of eggs to fertilization (Schroeder, 1978). It should be noted that the pace of stereociliary growth in response to trauma is much faster than that observed during embryonic development (Cotanche, 1987c; Tilney and DeRosier, 1986). This is not surprising since developmental growth requires coordinated control and continuous inductive interactions which take longer to accomplish. It is not clear whether the ectopic stereocilia which appear after noise trauma are newly formed or relocated from a previous site. While both options could be feasible, cells probably need energy for either one of these processes. Thus, we speculate that the reorganization of stereocilia bundles is an active process associated with stress response and/or repair mechanisms.

Elongated stereocilia and stress links appeared in short and tall hair cells in the basilar papilla. In the area of the tall hair cells, the tectorial membrane was not severely damaged, indicating that uncoupling from the tectorial membrane is not the signal for stereociliary elongation nor for stress link establishment. Moreover, stereocilia can clearly elongate and form contacts with other cells when the tectorial membrane covers their apical surfaces. For example, stereociliary growth under the tectorial membrane has been observed after noise exposure in guinea pigs and rabbits (Avinash et al., 1993; Engström et al., 1983; Raphael and Altschuler, 1992). It is interesting to note that the primary auditory sensory transducers (inner hair cells in mammals and tall hair cells in chicks) display stereociliary changes after noise exposure (Engström et al., 1983; Robertson, 1982), yet are less susceptible to degeneration after acoustic trauma.

Contacts between avian hair cells beneath the luminal surface have been thoroughly documented by Fischer et al. (1991), who also reviewed other evidence for cytoplasmic contacts between adjacent hair cells. While the function of the cytoplasmic contacts is not entirely clear, it is likely that trauma-induced expansion of supporting cells severs these intercellular con- tacts. It is possible that in order to secure intercellular communication, hair cells substitute for the loss of cytoplasmic contacts by creating stress links.

Adler et al. (1992) demonstrated that stereocilia bundle stiffness is not significantly altered in cells that survive a pure tone exposure, suggesting that these surviving cells maintain their normal physiological capacity. In future experiments it will be important to measure physiological correlates of surviving hair cells with altered stereociliary morphology. It would also be intcresting to detcrmine whether these cells participate in roles other than their normal role in transduction. For example, observations that hair cells lost due to acoustic trauma in the chick basilar papilla are replaced by new hair cells (Cotanche, 1987b) and subsequent evidence that supporting cells in the basilar papilla can proliferate and repopulate the epithelium after acoustic trauma (Raphael, 1992) raised interest in elucidating the signals that initiate and regulate the proliferative process in the avian auditory epithelium. It will be necessary to determine whether surviving hair cells play a role in mediating information relevant for survival under stress and regulation the regenerative process.

In conclusion, this study showed that hair cells that survive acoustic trauma in the chick basilar papilla may reorganize their stereocilia bundles. Stereociliary reorganization included elongation, supernumerary or ectopic bundles and apparent contacts between bundles of neighboring cells. These changes were observed at the end of a 4-h noise exposure in tall and short hair cells. We speculate that these structural changes in stereocilia reflect a response to stress conditions, and accordingly, we name the intercellular stereocilia links as 'stress links.'

\section{Acknowledgments}

This work was supported by a grant from the Deafness Research Foundation and an NIH Grant 1 RO1 DC01634. Thanks are due to Marcia M. Muller and Carlos A. Ricotti for valuable assistance in the photography work.

\section{References}

Adler, H.J., Kenealy, J.F., Dedio, R.M. and Saunders, J.C. (1992) Threshold shift, hair cell loss, and hair bundle stiffness following exposure to 120 and $125 \mathrm{~dB}$ pure tones in the neonatal chick. Acta Otolaryngol. (Stockh.) 112, 444-454.

Avinash, G.B., Nuttall, A.L. and Raphael, Y. (1993) 3-D analysis of F-actin in stereocilia of cochlear hair cells after loud noise exposure. Hear. Res. 67, 139-146.

Borg, E. and Engström, B. (1989) Noise level, inner hair cell damage, audiometric features, and equal-energy hypothesis. J. Acoust. Soc. Am. 86, 1776-1782. 
Cotanche, D.A. (1987a) Regeneration of the tectorial membrane in the chick cochlea following severe acoustic trauma. Hear. Res. 30, 197-206.

Cotanche, D.A. (1987b) Regeneration of hair cell stereociliary bundles in the chick cochlea following severe acoustic trauma. Hear. Res. 30, 181-196.

Cotanche, D.A. (1987c) Development of hair cell stereocilia in the avian cochlea. Hear. Res. 28, 35-44.

Cotanche, D.A., Saunders, J.C. and Tilney, L.G. (1987) Hair cell damage produced by acoustic trauma in the chick cochlea. Hear. Res. 25, 267-286,

Drenckhahn, D., Schafer, T. and Prinz, M. (1985) Actin, myosin and associated proteins in the vertebrate auditory and vestibular organs: Immunocytochemical and biochemical studies. In: D.G. Dresher (Ed.). Auditory Biochemistry. Charles C Thomas, Springfield, IL, pp. 317-335.

Engström, B., Flock, $\AA$. and Borg, E. (1983) Ultrastructural studies of stereocilia in noise-exposed rahbits. Hear. Res. 12, 251-264.

Fischer, F.P., Brix, J., Singer, I. and Miltz, C. (1991) Contacts between hair cells in the avian cochlea. Hear. Res. 53, 281-292.

Flock, A., Flock, B and Murray, E. (1977) Studies on the sensory hairs of receptor cells in the inner ear. Acta Otolaryngol. (Stockh.) $83,85-91$.

Flock, $\AA$. and Cheung, H.C. (1977) Actin filaments in sensory hairs of inner ear receptor cells. J. Cell Biol, 75, 339-343.

Hackney, C.M., Furness, D.N. and Sayers, D.L. (1988) Stereociliary cross-links between adjacent inner hair cells. Hear. Res. 34, 207-211.

Hall, A.L., Schlein, A, and Condeelis, J. (1988) Relationship of pseudopod extension to chemotactic hormone-induced actin polymerization in amoeboid cells. J. Cell Biochem. 37, 285-299.

Liberman, M.C and Beil, D.G. (1979) Hair cell condition and auditory nerve response in normal and noise-exposed cochleas. Acta Otolaryngol. (Stockh.) 88, 161-176.

Marsh, R.R., L. Xu, J.P. Moy, and J.C. Saunders (1990) Recovery of the basilar papilla following intense sound exposure in the chick. Hear. Res. 46, 229-238.

Raphael, Y. (1991) Damage to the tectorial membrane may protect chick hair cells from noise overstimulation. Hear. Res. 53, 173184.

Raphael, Y. (1992) Evidence for supporting cell mitosis in response to acoustic trauma in the avian inner ear. J. Neurocytol. 21. $663-671$.

Raphael, Y. (1993) Reorganization of the chick basilar papilla following acoustic trauma. J. Comp. Neurol. 330, 521-532.

Raphael, Y. and Altschuler, R.A. (1991) Modulation of cytoskeletal and junctional proteins during hair cell degeneration. Cell Motil. 18, 215-227.

Raphael, Y, and Altschuler, R.A. (1992) Early microfilament reorganization in injured auditory epithelia. Exper. Neurol. 115,32 36.

Robertson, D. (1982) Effects of acoustic trauma on stereocilia structure and spiral ganglion cell tuning properties in the guinea pig cochlea. Hear. Res. 7, 55-74.

Robertson, D. and Johnstone, B.M. (1980) Acoustic trauma in the guinea pig cochlea: early changes in ultrastructure and neural threshold. Hear. Res. 3, 167-179.

Santi, P.A. and Anderson, C.B. (1987) A newly identified surtace coat on cochlcar hair cells. Ilcar. Res. 27, 47-66.

Saunders, J.C., Cohen, Y.E. and Szymko, Y.M. (1991) The structural and functional consequences of acoustic injury in the cochlea and peripheral auditory system: a five year update. J. Acoust. Soc, Am. 90. 136-146.

Schroeder, T.E. (1978) Microvilli on sea urchin eggs: a second burst of elongation. Dev. Biol. 64, 42-46.

Slepecky, N. (1986) Overview of mechanical damage to the inner ear: noise as a tool to probe cochlear function. Hear. Res. 22, 307-321.

Slepecky, N. and Chamberlain, S.C. (1982) Distribution and polarity of actin in the sensory hair cells of the chinchilla cochlea. Cell Tissue Res. 224, 15-24.

Smith, S.M. (1991) Rapid indirect immunofluorescence of cultured cells facilitated by microwave heating. Biotchniques $11,340-34$.

Thorne, P.R., Gavin, J.B. and Herdson, P.B. (1984) A quantitative study of the sequence of topographical changes in the organ of Corti following acoustic trauma. Acta Otolaryngol. (Stockh.) 97. 69-81.

Thorne, P.R., Duncan, C.E and Gavin, J.B. (1986) The pathogenesis of stereocilia abnormalities in acoustic trauma. Hear. Res. 21. $41-49$.

Tilney, L.G., Saunders, J.C., Egelman, E. and DeRosier, D.J. (1982) Changes in the organization of actin filaments in the stereocilia of noise-damaged lizard cochleae. Hear. Res. 7, 181-197.

Tilney, L.G., Egelman, E.H., DeRosier, D.J. and Saunders, J.C. (1983) Actin filaments, stereocilia and hair cells of the bird cochlea. 11. The packing of actin filaments in the stereocilia and in the cuticular plate and what happens to the organization when stereocilia are bent. J. Cell Biol. 96, 822-834.

Tílney, L.G. and DeRosier, D.J. (1986) Actin filaments, stereocilia and hair cells of the bird cochlea. IV. How the actin filaments become organized in developing stereocilia and in the cuticular plate. Dev, Biol. 16, 119-129. 